\title{
CONOCIMIENTOS DE INFORMÁTICA DE LOS ALUMNOS DE LA FACULTAD DE CIENCIAS CONTABLES Y FINANCIERAS Y LA NECESIDAD DE DICHO CONOCIMIENTO PARA ENFRENTAR CON ÉXITO EL DESAFÍO LABORAL
}

Responsable: CPC Alexis Efrain Alpaca Cusicanqui

\section{RESUMEN}

El Contador siempre debe estar pendiente de las necesidades que demanda la sociedad, el mercado y la empresa; por ello, los estudiantes de Contabilidad deben recibir la enseñanza de informática conveniente y los docentes deben actualizar continuamente los conocimientos de Informática Contable que vierten en sus alumnos.

El presente trabajo de investigación ha sido efectuado para reconocer las necesidades Informático-Contables que actualmente tienen las empresas de la ciudad de Tacna, con la finalidad de promover que los docentes preparemos convenientemente a nuestros alumnos para que éstos puedan ubicarse rápidamente en el mercado laboral y logren un desempeño eficiente.

\section{INTRODUCCIÓN}

Hoy en dia, la competencia laboral exige una gran cantidad de requisitos para acceder a un puesto de trabajo. Además, con los adelantos tecnológicos es necesario incursionar en diversos temas de actualidad. Tal es el caso de la informática. Como debemos saber, los sistemas de cómputo se han introducido rápidamente en cualquier área, tanto en la medicina, la investigación científica, el gobierno, la educación, en el área militar, en la oficina e inclusive en el hogar. Por esa razón, todo estudiante universitario debe egresar de la Universidad preparado con conocimientos básicos relativos a la Informática, para ir a la par con la tecnologia informática en la sociedad en que vivimos.

Pero, ¿por qué el estudiante de la profesión contable debe saber Informática? Existen muchas razones, entre ellas la que los estudiantes de ahora deben preocuparse por aprender el nuevo mundo de la informática, para llegar a ser contadores que puedan desempeñar sus labores contables a través de los sistemas computacionales, obteniendo información veraz, clara, oportuna y ágil, ya que las computadoras y los paquetes contables ayudan a reducir significativamente el tiempo de procesamiento y presentación de la información financiera.

\section{OBJETIVOS}

Objetivo General : Precisar cuáles son los conocimientos necesarios sobre Informática que deben aprender los alumnos de la Facultad de
Ciencias Contables y Financieras, de acuerdo con las exigencias del mercado laboral.

Objetivos Especificos:

- Comprender la importancia de los conocimientos de informática para el profesional contable.

-Conocer las exigencias del mercado laboral, en cuanto a los conocimientos de informática que deben conocer los profesionales contables.

- Identificar las causas por las que los alumnos no perciben los conocimientos informáticos necesarios.

\section{MARCOTEORICO}

En el ámbito del desenvolvimiento de la profesión contable existen muchas influencias provenientes de diversos sectores del conocimiento, entre los cuales la informática ocupa un lugar preferencial. En tal sentido, los estudiantes de contabilidad deben recibir la preparación suficiente para poder desenvolverse en el mercado laboral.

La Contabilidad en general es una disciplina que ha sufrido profundas innovaciones en todos los sentidos: en cuanto a objetivos, a técnicas, a la incorporación de otras disciplinas científicas, al mercado de trabajo y de servicios, y finalmente, a haber entrado en competencia con otras rofesiones por el predominio de algunas áreas de trabajo, que anteriormente eran casi de su exclusividad,como la administración financiera, en donde ahora se ve precisada a competir con la rofesión del administrador.

La contablidad es una disciplina ampliamente aceptada por un núcleo social económico 
importantísimo que estimula a quienes la practican a adquirir, los conocimientos más avanzados acordes con la realidad.Para definir la profesión contable se requiere más que un campo de actuación, una teoría de la contabilidad que sirva de marco de referencia al desarrollo de la tecnología contable con la cual acudir a un mercado de trabajo y otro de servicios, que con el tiempo se han ampliado y continúan haciéndolo.

En la actualidad, el mundo ha cambiado y como profesionales contables no podemos abstraernos de estos cambios. La informática desde su aparición ha ido avanzando cada vez con mayor velocidad, a tal punto que el profesional contable imprescindiblemente debe adquirir los conocimientos informáticos que requiere la empresa para la aplicación de los distintos programas de aplicación contable financiera, así como, los conocimientos referidos a la instalación de sistemas de información en la empresa.Si bien es cierto que no se intenta que el profesional contable suplante a otros profesionales, debido a los requerimientos informáticos cada vez más especificos de las empresas, es necesario que en las aulas se imparta la enseñanza necesaria a los alumnos, para que al egresar se encuentren capacitados para trabajar en la creación de sistemas de información, para entender cómo funcionan los programas de aplicación contable y la utilización práctica de éstos, teniendo siempre en consideración la relación costo -beneficio, de acuerdo con la envergadura de la empresa.

\section{METODOLOGÍA DE LA INVESTIGACIÓN}

Tipo de Investigación :De acuerdo con su finalidad, será investigación aplicada porque permite realizar un análisis de los conocimientos de informática que reciben los estudiantes de la Facultad de Ciencias Contables y Financieras.Según su profundidad será descriptiva y explicativa, pues tratará de describir y explicar los conocimientos de informática de los estudiantes de la Facultad de Ciencias Contables y Financieras.

De acuerdo con su amplitud, será micro investigación, porque sólo abarca una población de una pequeña área geográfica.

De acuerdo con el enfoque, es especializada, porque se efectúa desde el punto de vista de la Ciencia Contable.

Diseño de Investigación:El diseño y método de la investigación es de tipo Diagnóstico Evaluativo, debide a que la naturaleza de las variables de estudio no permite utilizar un método experimental. El método Diagnóstico Evaluativo se ha utilizado porque además de describir los rasgos y caracteristicas de los conocimientos de Informática que se imparten en la Facultad de
Ciencias Contables y Financieras, se determinarán las carencias que podrian existir con las exigencias laborales por parte de las empresas.

Población y Muestra:La población está conformada por docentes y estudiantes de la Facultad de Ciencias Contables y Financieras de la Universidad Nacional Jorge Basadre Grohman, y por las empresas de la ciudad de Tacna. De la población de estudiantes, se ha obtenido una muestra aleatoria estratificada proporcional, esto quiere decir que se ha calculado una muestra aleatoria de la población total y posteriormente, en forma proporcional se calculó la muestra de cada año de estudios. Para hallar la muestra se utilizó el software Stats v1.1; la muestra estuvo constituida por 100 estudiantes de ambos sexos, que fue extraida de la población mencionada anteriormente, la que constituye aproximadamente el $59,88 \%$ de la población de alumnos de la Facultad.

Tabla 01:

\begin{tabular}{|c|c|c|}
\hline \multirow{2}{*}{$\begin{array}{c}\text { Ario de } \\
\text { Estudios }\end{array}$} & \multicolumn{2}{|c|}{ Estudiantes } \\
\cline { 2 - 3 } & oblación & Muestra \\
\hline $4^{\circ}$ & 81 & 49 \\
$5^{\circ}$ & 86 & 51 \\
Total & 167 & 100 \\
\hline
\end{tabular}

Se han considerado 10 docentes como muestra. Asimismo, se obtendrá información de 20 empresas de las diversas actividades económicas:

\section{Tabla 02:}

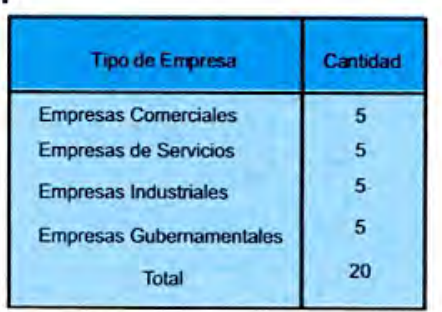

Técnicas de Recolección de Datos

Teniendo en cuenta que la encuesta es un conjunto de preguntas normalizadas dirigidas a una muestra representativa de la población o instituciones, con el fin de conocer estados de opinión o hechos especificos, para poder obtener datos significativos fue necesario elaborar tres cuestionarios de preguntas para aplicarlos a través de sendas encuestas a la muestra elegida de alumnos, a los docentes y a los empleados de las empresas. Los cuestionarios aplicados constan de preguntas abiertas preclasificados, de final abierto, tipo escala y dicotómicas.

Luego, se procedió a la calificación de las pruebas y se realizó el análisis estadistico de los resultados a través del paquete de análisis estadistico para la investigación en ciencias 
sociales, Stats (Versión 1.1), con el fin de comprobar la hipótesis planteada.

Cuadro 03: Conocimientos que reciben los alumnos de la Facultad de Ciencias Contables y Financieras de la UNJBG.

\begin{tabular}{|l|c|c|c|c|}
\hline \multirow{2}{*}{ Detalle } & \multicolumn{3}{|c|}{ Conocimiento } & \multirow{2}{*}{} \\
\cline { 2 - 5 } & Básico & Interm & Avanzado & Total \\
\hline Windows XP & 43 & 44 & 13 & 100 \\
\hline Office 2007 & 32 & 58 & 10 & 100 \\
\hline Linux & 19 & 0 & 0 & 19 \\
\hline Software educativo & 23 & 7 & 0 & 30 \\
\hline Software Contable & 16 & 78 & 6 & 100 \\
\hline
\end{tabular}

En el presente cuadro se observan los conocimientos de los alumnos de algunas áreas de la Informática, apreciando que lo que más conocen son los programas contables en un nivel intermedio, y lo que menos conocen es el sistema operativo Linux.

Cuadro 04: Conocimientos de programación que reciben los estudiantes de la Facultad de Ciencias Contables y Financieras de la UNJBG.

\begin{tabular}{|l|r|r|r|r|}
\hline \multirow{2}{*}{ Detalle } & \multicolumn{3}{c|}{ Conocimiento } & \multirow{2}{*}{ Total } \\
\cline { 2 - 4 } & Básico & Intermedio & Avanzado & \\
\hline $\begin{array}{l}\text { Algoritmos y } \\
\text { Diagramación }\end{array}$ & 88 & 12 & 0 & 100 \\
\hline Access & 16 & 0 & 0 & 16 \\
\hline Visual Basic & 19 & 0 & 0 & 19 \\
\hline Visual Fox Pro & 89 & 11 & 0 & 100 \\
\hline
\end{tabular}

En el presente cuadro se observan los conocimientos de programación que tienen los alumnos. Podemos ver que los tópicos que mas conocen son los algoritmos y el software Visual Fox con porcentajes de $88 \%$ y $89 \%$ respectivamente, pero sólo conocimientos básicos; también podemos decir que los programas que menos conocidos, y sólo a nivel básico, son Access y Visual Basic con 16\% y $19 \%$ respectivamente.

Cuadro 5: Conocimientos acerca de herramientas de Internet que reciben los alumnos de la Facultad de Ciencias Contables y Financieras de la UNJBG.

\begin{tabular}{|l|r|r|r|}
\hline \multirow{2}{*}{ Detalle } & \multicolumn{2}{|c|}{ Respuesta } & \multirow{2}{*}{ Total } \\
\cline { 2 - 3 } & $\mathrm{Si}$ & No & \\
\hline Correo Electrónico & 26 & 74 & 100 \\
\hline Office 2007 & 94 & 6 & 100 \\
\hline Páginas Web & 92 & 8 & 100 \\
\hline Software Educación & 69 & 31 & 100 \\
\hline Listas de Distribución & 90 & 10 & 100 \\
\hline Uso de Navegadores & 93 & 7 & 100 \\
\hline Aceleradores de Navegación & 87 & 13 & 100 \\
\hline Buscadores Especializados & 74 & 26 & 100 \\
\hline
\end{tabular}

En el presente cuadro se observa que las herramientas de Internet que se les ha enseñado a los alumnos y de las cuales se ha logrado el aprendizaje son Office, Páginas Web, uso de Listas de Distribución y uso de Navegadores, de las cuales saben los alumnos en porcentaje mayor al $90 \%$.
Cuadro 06: Capacitación de los alumnos para realizar un óptimo desempeño laboral, según opinión de los docentes.

\begin{tabular}{|l|c|c|}
\hline \multicolumn{1}{|c|}{ Respuesta } & Total & Porcentaje \\
\hline $\mathrm{Si}$ & 8 & 80 \\
\hline No & & \\
\hline Regularmente & 2 & 20 \\
\hline Total & 10 & 100 \\
\hline
\end{tabular}

En el presente cuadro se observa que el $80 \%$ de docentes opinan que los alumnos de la Facultad de Ciencias Contables y Financieras al egresar de las aulas universitarias se encuentran capacitados para desempeñar las funciones informáticas que les competen en los cargos contables

Cuadro 07: Verificación del cumplimiento de sus funciones laborales en las que tienen que utilizar sus conocimientos informáticos por parte de los egresados de la Facultad de Ciencias Contables y Financieras, según los empresarios.

\begin{tabular}{|l|c|c|}
\hline Respuesta & Total & Porcentaje \\
\hline $\mathrm{Si}$ & 13 & 65 \\
\hline No & 0 & 0 \\
\hline Sólo en parte & 7 & 35 \\
\hline Total & 20 & 100 \\
\hline
\end{tabular}

En el presente cuadro se observa que el $65 \%$ de los funcionarios de las empresas piensa que los egresados de la Facultad de Ciencias Contables y Financieras de Universidad Nacional Jorge Basadre Grohmann, que trabajan o han trabajado en sus empresas, han demostrado su capacitación en los aspectos informáticos requeridos.

\section{DISCUSIONDERESULTADOS}

El profesional contable, una vez egresado de las aulas universitarias y estando ya insertado en el campo laboral o buscando que insertarse en éste, muchas veces empieza a tener dificultades para cumplir con sus funciones, sobre todo cuando se tiene que utilizar tecnología informática de última generación y modernos paquetes programáticos para efectuar su trabajo.En este sentido, los egresados de la Facultad de Ciencias Contables y Financieras de la Universidad Nacional Jorge Basadre Grohmann, con los conocimientos básicos que adquieren en sus años de estudios, pueden desempeñarse en sus trabajos sin ningún problema. Sin embargo, en el país y aun en el extranjero existen mercados laborales mucho más exigentes que el tacneño; por esta razón es que se hace indispensable que las enseñanzas que se imparten en las aulas universitarias deben estar en constante renovación, afianzamiento, mejora y actualización y cambio cuando sea necesario, debido a la rapidez del avance de la Informática.

La finalidad de esta investigación no es pretender que el Contador Público tenga como 
objetivo desplazar a los profesionales de la Informática, los Ingenieros de Sistemas; por lo tanto, no se le puede exigir al egresado de la Facultad de Ciencias Contables y Financieras que sea conocedor de todos los aspectos relacionados con la Informática, lo que se pretende aclarar es que como Contadores debemos estar preparados y capacitados para entender claramente cómo se usan los programas o software informáticos y el hardware de la computadora para poder tener un mejor desenvolvimiento laboral.

\section{CONCLUSIONES}

1.En la situación actual en la que nos desarrollamos, la Informática es una disciplina que representa una herramienta integral en la realización de muchas tareas.

2.La computadora ha venido a facilitar muchas labores y a posibilitar el llevado a cabo de otras que anteriormente no se realizaban. Hoy en dia, el conocimiento de la informática nos brinda muchas oportunidades para mejorar nuestra calidad de vida tanto privada como profesional. Nuestro reto consiste en aprovechar las oportunidades para incrementar estos conocimientos, los cuales nos proporcionarán mayores posibilidades de éxito en el ámbito laboral. A los docentes de la Facultad de Ciencias Contables y Financieras, según la información obtenida, les falta mayor capacitación en cuanto a técnicas de enseñanza de las materias informáticas, lo cual va en contra del correcto aprendizaje por parte de los estudiantes.

3. Como conclusión final puedo mencionar que con los conocimientos que se imparten en la Facultad de Ciencias Contables y Financieras, los alumnos que se preocupan por obtener estos conocimientos estarán capacitados y listos para afrontar los requerimientos mínimos que exigen las empresas. La Facultad de Ciencias Contables y Financieras debe preocuparse constantemente en medir la relación que hay entre las exigencias del mercado laboral y mantener un currículo flexible que permita efectuar los cambios necesarios que permitan brindar en todo momento una alta capacitación a sus estudiantes, con la finalidad que éstos se mantengan en la vanguardia de la competencia laboral.

\section{RECOMENDACIONES}

Resulta importante que todos busquemos el aprendizaje de los conocimientos de Informática, no sólo para nuestro desarrollo profesional, sino también para nuestra vida particular. Nuestro reto consiste en aprovechar las oportunidades para incrementar estos conocimientos, los cuales nos proporcionarán mayores posibilidades de éxito en el ámbito laboral. Los estudiantes de Contabilidad deben preocuparse por profundizar sus conocimientos informáticos con la ayuda de los docentes, quienes deben capacitarse convenientemente a fin de volcar todos sus conocimientos para lograr elevar el nivel de los alumnos. La meta debe ser que los alumnos de tener conocimientos básicos, en el corto tiempo deben tener conocimientos intermedios y a mediano plazo, avanzados. Asimismo, se recomienda que debe ampliarse la lista de temas que se enseñan en las asignaturas de Informática.

Seria apropiado que la Facultad de Ciencias Contables y Financieras contrate especialistas en el proceso enseñanza aprendizaje, con la finalidad de capacitar a sus docentes en este campo. Este acto redundará en una mejor enseñanza por parte de los docentes y como consecuencia se obtendrá un mejor aprendizaje por parte de los alumnos.

La Facultad de Ciencias Contables y Financieras debe preocuparse constantemente en medir la relación que hay entre las exigencias del mercado laboral y mantener un currículo flexible que permita efectuar los cambios necesarios que permitan brindar en todo momento una alta capacitación a sus estudiantes, con la finalidad que éstos se mantengan en la vanguardia de la competencia laboral.

\section{BIBLIOGRAFIA}

Elizondo López, Arturo. 2003. Contabilidad Básica-Editorial Thompson, México.

Arroyo Morales, Angélica. 1997. Metodología de la investigación científica, Cusco, Facultad de Ciencias Contables y Financieras UNSAAC, Segunda Edición.

Hidalgo Ortega, Jesús. La tesis, 1991, Lima. Ediciones Fecat, Segunda Edición.

"Pedagogia universitaria en América Latina." Centro Interamericano de Desarrollo Académico www.cinda.cl.

"Gestión Docente Universitaria". Centro Interamericano de Desarrollo Académico www.cinda.cl.

Metodología y técnicas de investigación cientifica. PARDINAS, Felipe. México, Siglo XXI, 1985.

Técnicas de investigación y ejecución de Proyectos de Investigación Tecnológica Industrial. ITINTEC, 1985, Lima.

Técnicas de investigación social, Sierra Bravo, Madrid, Paraninfo, 1987.

Estadistica. Spiegel R., Serie McGRAWHILL BOOK 1979. 